E

EVALUAR
2017, Vol. 17, No. 1

ISSN $1667-4545$

Recuperado de www.revistas.unc.edu.ar

\title{
Traducción y validación de la versión abreviada del Freiburg Mindfulness Inventory (FMI-14)
}

\section{Translation and Validation of Short Version of Freiburg Mindfulness Inventory (FMI-14)}

\author{
Germán Pérez-Verduzco ${ }^{*} 1$, Francisco Augusto Laca-Arocena ${ }^{2}$ \\ 1. Universidad de Colima, México. \\ 2. Universidad de Colima, México.
}

Introducción

Método

Resultados

Discusión

Referencias

Recibido: 14/02/2017 Revisado: 03/03/2017 Aceptado: 22/03/2017

\section{Resumen}

Recientemente se ha incrementado el interés de la psicología y otras ciencias de la salud por el concepto de Mindfulness. La evaluación de este constructo es cada vez más frecuente en el ámbito clínico para diseñar intervenciones enfocadas en mejorar la salud física y/o psicológica. En este trabajo se ha traducido y validado el Freiburg Mindfulness Inventory, en su versión breve (FMI-14). La muestra estuvo conformada por 200 personas de nacionalidad mexicana pertenecientes tanto a población universitaria como no universitaria. El instrumento se analizó a través de varias técnicas estadísticas, entre ellas un análisis factorial exploratorio (AFE) por medio del cual se identificaron cuatro componentes del Mindfulness, aquí denominados: atención, autocontrol, aceptación y autopercepción. Posteriormente, mediante un análisis factorial confirmatorio (AFC) se encontró que este modelo presenta un buen ajuste a los datos (CFI $=.93 ; \mathrm{GFI}=.93 ; \mathrm{AFGI}=.89 ; \mathrm{SRMR}=.05 ; \mathrm{RMSEA}=$ .04). Los resultados también indicaron que la versión en español del FMI-14 es confiable $(\alpha=.80)$ y válida para evaluar Mindfulness.

Palabras clave: Mindfulness, bienestar subjetivo, análisis factorial exploratorio, análisis factorial confirmatorio, FMI-14

\begin{abstract}
In recent years, interest in studying Mindfulness and its relationship with subjective well-being has increased. In addition, evaluation of this construct is becoming more common in clinical practice, in order to design interventions to enhance physical and psychological health. In this study, the short version of Freiburg Mindfulness Inventory (FMI-14) has been translated and validated. The study sample consisted of 200 Mexican individuals, from both university and non-university settings. The scale was analyzed with various statistical methods, including exploratory factor analysis (EFA) and confirmatory factor analysis (CFA). Four components of Mindfulness, hereby called attention, self-control, acceptance, and self-perception were found by using EFA. Subsequently, by using CFA, we found that this model had a good fit $(\mathrm{CFI}=.93$; $\mathrm{GFI}=.93$; $\mathrm{AFGI}=.89 ; \mathrm{SRMR}=.05 ; \mathrm{RMSEA}=.04) . \mathrm{Fi}-$ nally, our results show that our instrument is reliable $(\alpha=$ .80) and valid to assess Mindfulness in Spanish-speaking population.
\end{abstract}

Keywords: Mindfulness, subjective well-being, exploratory factor analysis, confirmatory factor analysis, FMI14 


\section{Introducción}

Mindfulness: conciencia y atención plena al presente

Tradicionalmente, así como la medicina, la psicología se ha interesado casi exclusivamente en la enfermedad, la disfunción y el dolor. El interés de la psicología académica por los estados de bienestar no aparecería hasta las últimas décadas del siglo pasado, cuando surgieron conceptos como bienestar subjetivo (Diener, 1984), optimismo aprendido (Seligman, 1992) o felicidad objetiva (Kahneman, 2003). La relación entre bienestar subjetivo y conciencia del momento presente había recibido hasta ese momento poca atención empírica. Sin embargo, recientemente existe un mayor número de investigaciones sobre la conciencia plena, también denominada Mindfulness (Brown, Ryan, \& Creswell, 2007; Jiménez, Niles, \& Park, 2010; Kabat-Zinn, 2013).

Actualmente, la conciencia plena se considera una de las funciones de la mente que promueve el bienestar psicológico o subjetivo. Esta práctica, realizada intencionadamente y desprovista de contextos religiosos, ha sido popularizada en occidente como Mindfulness, término inglés equivalente a la expresión "sati", de la lengua pali, que describe conciencia y atención (Siegel, Germer, \& Olendski, 2009). En el presente estudio mantenemos el término inglés entendiendo que está ampliamente aceptado y divulgado en nuestra lengua (Simon, 2013).

Inicialmente, las investigaciones sobre Mindfulness sólo eran relevantes en el ámbito de la psicología budista. Sin embargo, recientemente, este constructo ha tomado cada vez más importancia dentro de las ciencias de la salud (Kohls, Sauer, \& Walach, 2009) y en el estudio de la psicología social y de la personalidad (Herndon, 2008). En concreto, la psicología se interesó en el Mindfulness por su capacidad reguladora de la emoción (Baer, 2003) y como un moderador de las respuestas exageradas al entorno, derivadas del estrés y la ansiedad (Astin, 1997; Jiménez et al., 2010). Practicantes habituales de Mindfulness afrontan con eficacia dichos estados, manejan mejor sus emociones e incluso presentan mejoras en cuanto a sus defensas orgánicas (Kabat-Zinn, 2013). Asimismo, hay evidencia de que la atención plena brinda flexibilidad a las respuestas conductuales, incrementando la capacidad del individuo para responder de acuerdo a valores y objetivos, en lugar de hacerlo de forma automática o impulsiva (Brown et al., 2007).

Además de ser un medio de regulación emocional, el Mindfulness disminuye la falta de aceptación de ciertas experiencias y la rumiación, las que contribuyen al desarrollo de cuadros depresivos, por lo que su práctica ha sido adoptada como recurso en algunas terapias cognitivas frente a la depresión o el malestar psicológico (Hayes \& Feldman, 2004; Hernando-Mazón, 2012; Segal, Williams, \& Teasdale, 2012). Se ha observado que la intensidad de la tendencia a rumiar está relacionada, en pacientes depresivos, con procesos más severos y prolongados. Debido a este fenómeno, los terapeutas de orientación cognitiva están comenzando a afrontar dichas conductas mediante la incorporación de la meditación como otro recurso terapéutico (André, 2010). Por ejemplo, desde hace años, el Centro Médico de la 
Universidad de Massachusetts está aplicando y ha popularizado un programa de ocho semanas para la reducción del estrés basado en la aplicación de Mindfulness (llamado MBSR, por sus siglas en inglés; véase Kabat-Zinn, 2013). Existen estudios que por medio de metaanálisis han evaluado la efectividad clínica del programa y han encontrado efectos significativos tanto en la reducción del estrés (Grossman, Niemann, Schmidt, \& Walach, 2004) como en la atenuación de ciertos síntomas de depresión y ansiedad (Hofmann, Sawyer, Witt, \& Oh, 2010). Otros trabajos que encontraron que el Mindfulness ayuda a disminuir la ansiedad o el estrés son los realizados por Pérula-Jiménez (2016), Arias (2015), García-García (2015), Marzo-Andreu (2015), Rubio-Galtier (2015) y Torné-Novell (2013), en distintas universidades españolas.

Además de sus efectos positivos sobre el estrés, los trastornos de ansiedad y el estado de ánimo, también hay evidencia sobre los beneficios del Mindfulness en áreas que hasta hace poco no habían sido consideradas. En particular, una investigación reciente sobre el estado del arte de las IBM (Intervenciones Basadas en Mindfulness), encontró que las mismas resultaban eficaces en el tratamiento de pacientes con dolor crónico, cáncer, psicosis, y en personas que padecen adicciones (Hervás, Cebolla, \& Soler, 2016). Asimismo, algunos estudios informan una correlación positiva entre rendimiento académico y Mindfulness (Alfaro-Bouroncle, 2016); e incluso, en otros estudios el Mindfulness se propone como una estrategia eficaz para casos de abuso sexual infantil (Feligreras-Alcalá, 2014) o para intervenciones en alumnos con discapacidad intelectual (De la Calle-Ortega,
2016). Así, toda esta evidencia empírica ha provocado un aumento en el interés por incorporar esta práctica a los tratamientos médicos y psicológicos, lo que convierte al Mindfulness en un constructo cada vez más valorado en el ámbito científico.

\section{Medición de Mindfulness}

Si bien la uni o multidimensionalidad del Mindfulness todavía es objeto de discusión en la literatura sobre el tema (Soler, 2014); Brown y Ryan (2003), autores de la Mindfulness Attention Awareness Scale (MAAS), describen el Mindfulness como una única dimensión de atención o conciencia de lo que está ocurriendo en el momento presente, sin considerar otros atributos como la aceptación o la empatía, que frecuentemente se asocian al constructo. Esta escala fue traducida al español por Barajas y Garra (2014) y puesta a prueba en una muestra compuesta de pacientes con trastornos de ansiedad y depresión, en otra proveniente de población general y en una última compuesta de estudiantes. La adaptación al español del MAAS también mostró una estructura unifactorial y propiedades psicométricas adecuadas, entre ellas una elevada consistencia interna ( $\alpha$ $=.88$ ). Otro instrumento que reporta unidimensionalidad es el Child and Adolescent Mindfulness Measure (CAMM; Greco, Baer, \& Smith, 2011), que permite evaluar Mindfulness de manera confiable en niños y adolescentes entre 9 y 18 años $(\alpha=.84)$. La adaptación al español de la CAMM también ha mostrado ser válida y confiable (Turanzas, 2013); sin embargo, a di- 
ferencia del instrumento original, los datos obtenidos en su adaptación apoyan la hipótesis de una estructura bifactorial del constructo.

Otros estudios también proponen la bidimensionalidad del Mindfulness; en concreto, un factor atencional de conciencia del presente y otro actitudinal sobre su aceptación. En esta línea se encuentran la Toronto Mindfulness Scale (TMS; Lau et al., 2006) y la Philadelphia Mindfulness Scale (PMS; Cardaciotto, Herbert, Forman, Moitra, \& Farrow, 2008). Aunque estas escalas han sido utilizadas principalmente en investigaciones dirigidas a analizar los efectos de la meditación, la PMS ha sido traducida recientemente al español y ha sido validada en estudiantes y pacientes psiquiátricos españoles que no realizan habitualmente esta práctica (Tejedor et al., 2014), lo que indicaría que ésta puede utilizarse tanto en poblaciones generales como clínicas. Cabe mencionar que en la adaptación de esta escala también se encontró una estructura bidimensional.

Por otro lado, hay otros instrumentos que indican la existencia de varias dimensiones. Un ejemplo es la Cognitive and Affective Mindfulness Scale (CAMS; Feldman, Hayes, Kumar, Greeson, \& Laurenceau, 2007), que mide cuatro aspectos del Mindfulness: atención, alerta, enfoque en el presente y aceptación de las situaciones. Otro ejemplo es el Five Facets of Mindfulness Questionnaire (FFMQ; Baer, Smith, Hopkins, Krietemeyer, \& Toney, 2006), que plantea cinco componentes: observación, descripción, actuar conscientemente, sin juicio de la experiencia interna y sin reactividad ante la experiencia interna. La FFMQ ha sido traducida al español (Cebolla et al., 2012), y ha demostra- do ser una escala válida tanto en la población española en general como en el ámbito clínico. A su vez, ha sido validada en estudiantes universitarios chilenos, donde ha demostrado ser un instrumento psicométrico adecuado (Schmidt \& Vinet, 2015).

Una escala que otorga peso a otros aspectos como la aceptación o el estado de alerta, aunque sin proponer la existencia de multidimensionalidad, es el Inventario Friburgo de Mindfulness (Walach, Buchheld, Buttenmüller, Kleinknecht, \& Schmidt, 2006). La versión completa del FMI (por sus siglas en inglés) es una escala de 30 ítems que suele aplicarse a personas que habitualmente practican la meditación (Trousselard et al., 2010); no obstante, existe una versión corta denominada FMI-14, diseñada para individuos que no están familiarizados con la meditación. En principio, todas las personas tienen la capacidad de atender al momento presente y ser conscientes de él, aunque esta habilidad mental varía, y puede verse estimulada o debilitada por diversos factores. A esos estados espontáneos de conciencia plena del presente quizás fuera pertinente describirlos como "disposición al Mindfulness" (Jiménez et al., 2010). La investigación sobre Mindfulness en este contexto, en el cual los estados de atención plena surgen espontáneamente, como un proceso natural y no buscado deliberadamente como sucede con la meditación, resulta muy escasa. Por tal motivo, y debido a que actualmente no hay una adaptación del FMI-14 al español, en este trabajo hemos traducido y validado el instrumento. 


\section{Método}

Participantes

La muestra estuvo conformada por 200 personas mexicanas: $53.5 \%$ hombres $(n=107)$ y $46.5 \%$ mujeres $(n=93)$, con un rango de edad de 17 a 35 años, una media igual a $22.16(\mathrm{DE}=$ 3.21), y un valor modal y una mediana iguales a 21. No se observaron diferencias significativas en cuanto al género de los sujetos $\left(t_{[1]}=.78, p=\right.$ .43, $\mathrm{N}=200$, dos colas), ni asociaciones entre la edad y su nivel de Mindfulness $(r=.10, p=.15$, $\mathrm{N}=200$ ). Aunque se utilizó un muestreo por conveniencia, se procuró que el estudio incluyera tanto una población universitaria como una no universitaria. Para ello, solamente el 50\% (100 cuestionarios) se distribuyó entre estudiantes de la Universidad de Colima, de forma grupal y en las instalaciones universitarias; mientras que el otro 50\% se distribuyó individualmente en ciertos lugares públicos de la ciudad.

\section{Instrumento}

El FMI-14 es una escala confiable $(\alpha=$ .86) que captura diversos aspectos relevantes del Mindfulness. Al contrastarla con la versión larga (FMI-30), se encontró un alto nivel de correlación $(r=.95)$, lo que indica que posee adecuadas propiedades psicométricas (Walach et al., 2006). Cabe mencionar que el instrumento ha sido traducido y validado previamente en alemán (Heidenreich, Ströhle, \& Michalak, 2006), inglés (Leigh, Bowen, \& Marlatt, 2005) y francés (Trousselard et al., 2010). Para el presente trabajo, cada uno de los autores (ambos bilingües) realizó su propia traducción del instrumento. Posteriormente, se conformó un solo cuestionario a partir del consenso entre ambos investigadores. A continuación se le solicitó a un experto en el idioma (también bilingüe) que elaborara su propia traducción y la comparara con el instrumento consensuado. De tal manera que, del análisis de este segundo consenso, surgió la versión final del cuestionario.

\section{Análisis de datos}

Primeramente, se analizó la distribución de los ítems a través de diversas medidas de dispersión. Posteriormente, se llevó a cabo un análisis factorial exploratorio (AFE) para identificar la estructura subyacente en la matriz de datos. Para conocer el nivel de adecuación muestral y saber si los datos eran factorizables, se aplicó el coeficiente KMO (Kaiser, 1970) y también la prueba de esfericidad de Bartlett (1950). La cantidad de factores a retener se determinó revisando tanto el criterio de Kaiser (1960) como el de Cattell (1966), y se recurrió al método de componentes principales y a la rotación varimax a fin de optimizar la solución por columna, ya que éstos son los más utilizados para esta clase de análisis (Costello \& Osborne, 2005). Para la inclusión de los ítems en alguno de los factores se decidió que la carga mínima fuera .40 , punto de corte sugerido para muestras inferiores a 300 casos (Williams, Onsman, \& Brown, 2010). La consistencia interna de la escala se midió con el coeficiente alfa de Cronbach (1951).

Posteriormente, se contrastaron las estructuras obtenidas en el AFE por medio de un aná- 
lisis factorial confirmatorio (AFC). La bondad de ajuste de los modelos resultantes se evaluó con diversos indicadores: $\chi^{2}, \chi^{2} / g l$, CFI, GFI, AGFI, SRMR y RMSEA. A través de la valoración conjunta de estos índices fue posible establecer la validez de los modelos presentados. De acuerdo a Byrne (2010), son indicadores de buen ajuste un valor de SRMR igual o menor que .05, CFI, GFI y AGFI cercanos a 1, y un RMSEA igual o menor que .05. Sin embargo, el ajuste del modelo sería aceptable si los valores CFI, GFI y AGFI se acercaran a 90 (Bentler, 1990, 1992; Byrne, 2010) y el índice RMSEA fuera igual o menor que $.06(\mathrm{Hu} \&$ Bentler, 1999). Finalmente, un valor $\chi^{2} / g l$ inferior a 2 es indicativo de un ajuste excelente (Escurra-Mayaute \& Salas-Blas, 2014).

El software utilizado para el análisis fue la versión 22 de SPSS (Statistical Package for the Social Sciencies; IBM, 2013) y el programa AMOS 19.0 (Arbuckle, 2014).

\section{Consideraciones éticas}

La aplicación del instrumento se llevó a cabo de acuerdo a los aspectos de confidencialidad y consentimiento informado considerados

Tabla 1

Matriz de componentes rotados.

\begin{tabular}{rcccc}
\hline \multirow{2}{*}{ Ítems } & \multicolumn{4}{c}{ Factores } \\
\cline { 2 - 5 } & $\mathbf{1}$ & $\mathbf{2}$ & $\mathbf{3}$ & $\mathbf{4}$ \\
\hline 6 & .69 & .19 & .00 & .17 \\
5 & .63 & .22 & .31 & -.12 \\
1 & .55 & -.11 & .40 & .36 \\
4 & .51 & .37 & -.02 & .31 \\
7 & .48 & .16 & .28 & .26
\end{tabular}

por el Código Ético del Psicólogo (Sociedad Mexicana de Psicología, 2007).

\section{Resultados}

Al revisar los niveles de asimetría y curtosis de cada uno de los ítems se encontró normalidad univariante; es decir, no se presentaron datos que distorsionaran la información obtenida con la aplicación del instrumento. El análisis factorial exploratorio generó cuatro factores (ver Tabla 1) que explican hasta el 55.11\% de la variabilidad. La rotación convergió en 15 iteraciones; el estadístico KMO arrojó un valor de .83, y la prueba de esfericidad de Bartlett resultó significativa $(p<.05)$. Estos resultados indican que la solución es adecuada para la muestra y que la utilización del modelo factorial es pertinente para el conjunto de datos analizados. Los múltiples factores resultantes podrían denominarse tentativamente: Atención (1), Autocontrol (2), Aceptación (3) y Autopercepción (4). Por otra parte, el gráfico de sedimentación sugiere la presencia de un factor dominante en la estructura matricial. Además, al solicitar una solución unifactorial se observa que todos los ítems presentan cargas entre .38 y .61 (ver Tabla 2). El alfa de Cronbach fue de .80, lo que indica buena consistencia interna del instrumento.

Tabla 2

Cargas de los ítems en una solución unifactorial.

\begin{tabular}{cc}
\hline Ítems & Saturaciones \\
\hline 1 & .61 \\
6 & .61 \\
7 & .59 \\
9 & .58 \\
12 & .58
\end{tabular}




\begin{tabular}{rrrrr}
\hline \multirow{2}{*}{ Ítems } & \multicolumn{4}{c}{ Factores } \\
\cline { 2 - 5 } & $\mathbf{1}$ & $\mathbf{2}$ & $\mathbf{3}$ & $\mathbf{4}$ \\
\hline 11 & .11 & .72 & .07 & -.03 \\
10 & .16 & .70 & .05 & .11 \\
9 & .27 & .57 & .33 & -.10 \\
8 & .32 & -.07 & .72 & -.04 \\
12 & .13 & .20 & .67 & .04 \\
14 & -.21 & .34 & .54 & .45 \\
13 & .04 & .39 & .45 & .23 \\
2 & .13 & -.07 & .09 & .76 \\
3 & .19 & .09 & -.00 & .72 \\
\hline
\end{tabular}

Para corroborar los resultados del AFE y comparar las diferencias entre los dos criterios de factorización utilizados, éstos se contrastaron mediante AFC. En el modelo unidimensional, 12 de los 14 ítems tuvieron cargas de al menos .40, siendo la excepción los ítems 2 y 3 ,

\begin{tabular}{cc}
\hline Ítems & Saturaciones \\
\hline 5 & .56 \\
4 & .55 \\
13 & .55 \\
14 & .52 \\
8 & .51 \\
10 & .51 \\
11 & .45 \\
3 & .43 \\
2 & .38 \\
\hline
\end{tabular}

con valores de .35 y .37 , respectivamente (ver Figura 1). Por otro lado, en el modelo de cuatro factores relacionados, las cargas de los ítems oscilaron entre .49 y .67 , aunque la correlación entre los factores 2 y 4 fue cercana a cero (Figura 2).

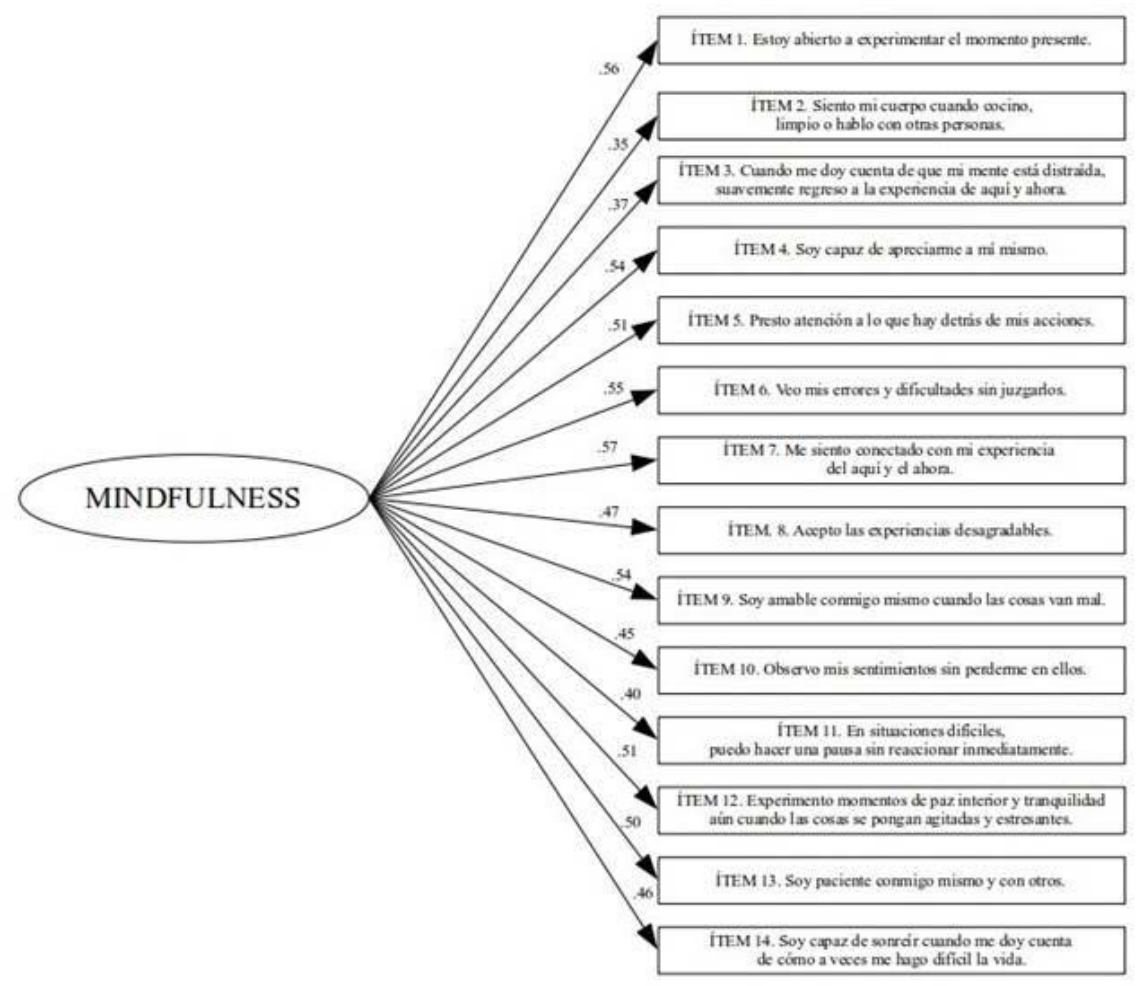

Figura 1

Modelo de un factor del FMI-14: Análisis factorial confirmatorio de la solución unidimensional del FMI-14 (N = 200). Los rectángulos indican las variables observadas, en este caso los 14 ítems de la escala; mientras que el óvalo representa la variable latente o no observada, es decir el constructo de Mindfulness. Los valores sobre las flechas indican los pesos de regresión estandarizados. 


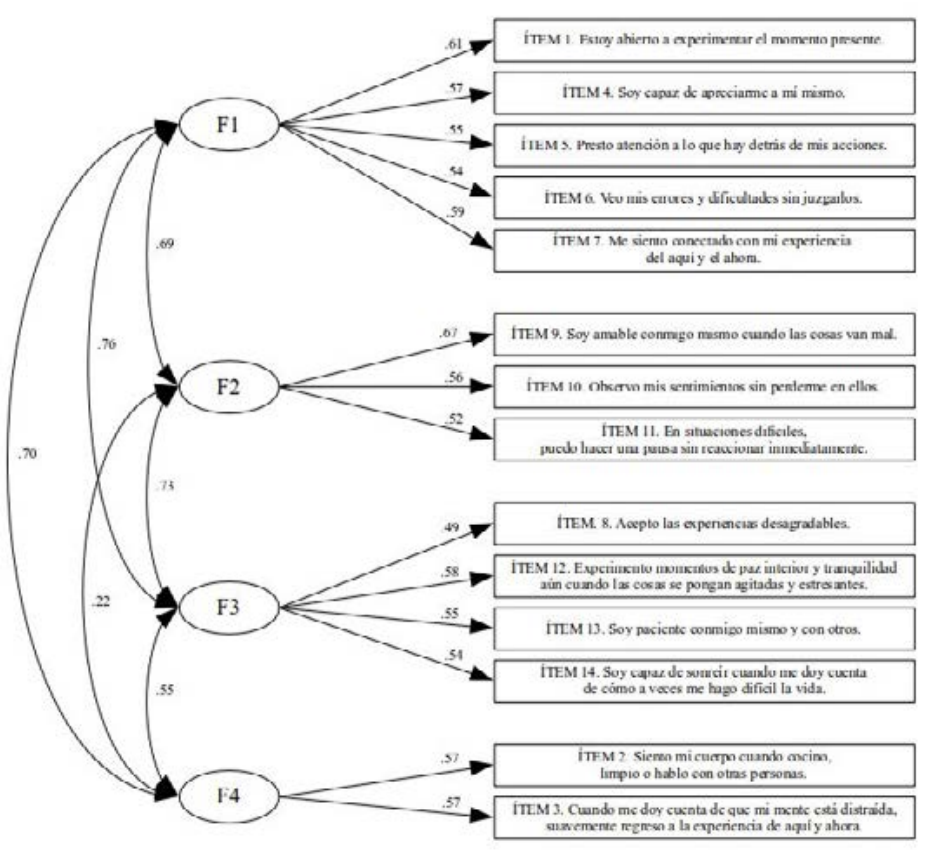

Figura 2

Modelo de cuatro factores relacionados del FMI-14: Análisis factorial confirmatorio de la solución de cuatro factores relacionados del FMI-14 ( $\mathrm{N}=200)$. Los rectángulos indican las variables observadas, en este caso los 14 ítems de la escala; mientras que el óvalo representa la variable latente o no observada, es decir el constructo de Mindfulness. Los valores sobre las flechas indican los pesos de regresión estandarizados.

Finalmente, al revisar diversos indicadores de bondad de ajuste en ambos modelos se encontró que el de cuatro factores relacionados se ajustaba mejor a los datos (ver Tabla 3).
Friburgo de Mindfulness (IFM-14) es un instrumento confiable, dado que presenta buena consistencia interna según lo evaluado mediante el coeficiente alfa de Cronbach. Por otra parte, se verificó que los ítems presentan niveles bajos de

Tabla 3

Indicadores de bondad de ajuste de los modelos de 1 y 4 factores del FMI-14.

\begin{tabular}{lcccccccc}
\hline \multicolumn{1}{c}{ Modelo } & $\chi^{2}$ & $\boldsymbol{g} \boldsymbol{l}$ & $\chi^{2} / \boldsymbol{g} \boldsymbol{l}$ & CFI & GFI & AGFI & SRMR & RMSEA \\
\hline 1 Factor & 155.98 & 77 & 2.02 & .84 & .89 & .85 & .06 & .07 \\
4 Factores relacionados & 104.77 & 71 & 1.47 & .93 & .93 & .89 & .05 & .04
\end{tabular}

Nota. Como ya se indicó en el apartado Método, los criterios para obtener un ajuste aceptable son: CFI, GFI y AGFI cercanos a $.90, \mathrm{SRMR} \leq .05, \mathrm{RMSEA} \leq .06$ y $\chi^{2} / g l<2$.

\section{Discusión}

El principal objetivo de esta investigación fue traducir y validar el FMI-14. Los análisis estadísticos realizados indican que el Inventario asimetría y curtosis, lo cual indica ausencia de sesgo en la distribución de los datos.

En cuanto a los resultados sobre la estructura de los datos, la naturaleza unidimensional del Mindfulness no resulta muy clara. Aunque 
esta situación ya se ha presentado en otras investigaciones (Baer et al., 2006; Feldman et al., 2007; Kohls et al., 2009; Trousselard et al., 2010), el número de dimensiones identificadas en este trabajo fue dependiente principalmente del criterio de factorización: mientras que con el criterio de Kaiser (1960) se obtuvo una solución de cuatro factores, con el de Cattell (1966) se encontró una dimensión principal. Por otra parte, al contrastar ambas alternativas en el AFC, se aprecia que el modelo de cuatro factores se ajusta mejor a los datos, pues éste presenta índices de bondad de ajuste más elevados que el modelo unifactorial. Así, el Mindfulness podría considerarse como un constructo compuesto por cuatro aspectos, aquí denominados: atención, autocontrol, aceptación y autopercepción. Sin embargo, antes de extraer conclusiones, algunas cuestiones merecen ser analizadas con mayor profundidad.

Si bien el modelo de cuatro factores representa mejor la estructura que subyace a los datos, cuando se evalúa la matriz de componentes rotados se observa que algunos ítems poseen saturaciones de al menos .40 para más de un factor. Además, si se hubiera establecido .30 como punto de corte, que es la práctica más común en los análisis factoriales (Lloret-Segura, Ferreres-Traver, Hernádez-Baeza, \& Tomás-Marco, 2014), se habría encontrado que varios ítems saturan en dos, o incluso hasta en tres factores. Por otro lado, en la solución unifactorial se observa que todos los ítems de la escala presentan saturaciones aceptables (entre .38 y .61), lo cual contribuye a corroborar la hipótesis de unidimensionalidad; y si a esto se le agrega el hecho de que la mayoría de las correlaciones entre los factores son elevadas, la descomposición del constructo en varios elementos se hace poco recomendable (Walach et al., 2006).

Cualquier investigación, independientemente de si está enfocada en realizar aportes a nivel teórico o empírico, pierde todo sentido sin la posibilidad de una aplicación posterior del conocimiento que genera. Por lo tanto, no debe olvidarse que uno de los principales objetivos de contar con instrumentos para evaluar Mindfulness es precisamente que éstos puedan utilizarse en intervenciones clínicas o educativas. En ese sentido, puede establecerse que la concepción unidimensional del Mindfulness, aunque menos exacta, es estadísticamente válida (Kohls et al., 2009) y tiene mayor aplicabilidad en la práctica, pues coincidimos con Barajas y Garra (2014) en que una estructura unifactorial es más conveniente para detectar relaciones más definidas con otras variables.

La conclusión anterior no implica abandonar los esfuerzos por comprender mejor la naturaleza del Mindfulness, o su relación con procesos psicológicos como el bienestar, la atención o la senso-percepción. Para tales casos, quizás resulte más útil investigar desde una perspectiva multidimensional del constructo (Trousselard et al., 2010). Sin embargo, en lo que se refiere a su uso como variable reguladora o mediadora del estrés, ansiedad u otros problemas de salud, consideramos más adecuada la perspectiva unidimensional. Además, si se deseara mejorar la homogeneidad del instrumento, podrían retirarse los ítems 2, 3 y 11 , ya que análisis estadísticos previos sobre su funcionamiento reflejan que eliminando estos reactivos el modelo unidimensional explica un mayor porcentaje de la 
variablidad (Laca-Arocena, Pérez-Verduzco, \& Vargas-Valencia, 2016).

Se recomienda la aplicación de esta escala en grupos de sujetos con características específicas; por ejemplo, en grupos de individuos familiarizados con el Mindfulness, que practiquen algún tipo de meditación, o de sujetos con alguna enfermedad o tratamiento particular. De este modo podría compararse el funcionamiento del instrumento en distintos contextos. También se sugiere que futuras investigaciones exploren las relaciones entre puntuaciones obtenidas con esta escala y otras similares, como la versión en español del MAAS (Mindful Attention Awareness Scale; Soler et al., 2012), la FFMQ (Five Facets of Mindfulness Questionnaire; Cebolla et al., 2012), o la SBC (Scale of Body Connection; Quezada-Berumen, González-Ramírez, Cebolla, Soler, \& García-Campayo, 2014), ya que una limitación importante de este trabajo es la falta de contraste con otros instrumentos que midan constructos similares o relacionados en la teoría. Esta sería otra forma de evaluar la validez de constructo de tales instrumentos.

Otra limitación de este trabajo es que la información recabada se obtuvo a través de un cuestionario, el cual, como otros métodos de autoinforme, no está exento del sesgo ocasionado por la deseabilidad social. Por ello, sería recomendable incorporar metodologías complementarias, como la evaluación de tareas basada en el rendimiento (Baer, Walsh, \& Lykins, 2009). Asimismo, futuras investigaciones podrían incluir un mayor número de variables sociodemográficas u otras características individuales de los participantes; de esta manera podría explorarse más profundamente la naturaleza del
Mindfulness como rasgo más que como actividad.

Finalmente, consideramos que el Mindfulness es un concepto con mucho potencial dentro de los ámbitos de la psicología positiva, la psicología clínica, la psiquiatría y en el área de salud en general, y en campos más específicos, como las terapias de tercera generación o las terapias contextuales. Sin duda, la investigación sobre este constructo aún puede aportar nuevos conocimientos sobre la comprensión de los procesos mentales, emocionales y la sensación de bienestar.

\section{Referencias}

Alfaro-Bouroncle, M. (2016). Mindfulness, flow y rendimiento académico en estudiantes universitarios (Tesis de grado). Recuperado de http://tesis.pucp. edu.pe/repositorio/handle/123456789/7467

André, C. (2010). Los estados de ánimo, el aprendizaje de la serenidad. Barcelona: Kairós.

Arbuckle, J. L. (2014). Amos (Versión 23.0) [Software]. Chicago: IBM SPSS.

Arias, Y. (2015). Eficacia de la aplicación de la terapia mindfulness para reducir los síntomas de ansiedad en personas adultas de Atención Primaria (Tesis de grado). Universidad del País Vasco, España.

Astin, J. A. (1997). Stress reduction through mindfulness meditation. Psychotherapy and Psychosomatics, 66(2), 97-106. doi: 10.1159/000289116

Baer, R. A. (2003). Mindfulness training as a clinical intervention: A conceptual and empirical review. Clinical Psychology: Science and Practice, 10(2), 125-143. doi: 10.1093/clipsy.bpg015

Baer, R. A., Smith, G. T., Hopkins, J., Krietemeyer, J., \& Toney, L. (2006). Using self-report assessment methods to explore facets of mindfulness. Assessment, 13(1), 27-45. doi: 10.1177/1073191105283504

Baer, R., Walsh, E., \& Lykins, E. (2009). Assessment of 
mindfulness. En F. Diddona (Ed.), Clinical Handbook of Mindfulness (153-168). New York: Springer.

Barajas, S., \& Garra, L. (2014). Mindfulness and psychopathology: Adaptation of the Mindful Attention Awareness Scale (MAAS) in a Spanish sample. Clínica y Salud, 25(1), 49-56. doi: 10.5093/ $\mathrm{cl} 2014 \mathrm{a} 4$

Bartlett, M. S. (1950). Tests of significance in factor analysis. British Journal of Psychology, 3(2), 7785. doi: 10.1111/j.2044-8317.1950.tb00285.x

Bentler, P. M. (1990). Comparative fit indexes in structural models. Psychological Bulletin, 107, 238-246. doi: 10.1037/0033-2909.107.2.238

Bentler, P. M. (1992). On the fit of models to covariances and methodology to the Bulletin. Psychological Bulletin, 112(3), 400-404. doi: 10.1037//00332909.112.3.400

Brown, K. W., \& Ryan, R. M. (2003). The benefits of being present: Mindfulness and its role in psychological well-being. Journal of Personality and Social Psychology, 84(4), 822-848. doi: 10.1037/00223514.84.4.822

Brown, K. W., Ryan, R. M., \& Creswell, J. D. (2007). Mindfulness: Theoretical foundations and evidence for its salutary effects. Psychological Inquiry, 18(4), 211-237. doi: 10.1080/10478400701598298

Byrne, B. M. (2010). Structural equation modeling with AMOS: Basic concepts, applications, and programming. New York: Routledge, Taylor \& Francis Group.

Cardaciotto, L., Herbert, J. D., Forman, E. M., Moitra, E., \& Farrow, V. (2008). The assessment of present-moment awareness and acceptance: The Philadelphia Mindfulness Scale. Assessment, 15(2), 204-223. doi: 10.1177/1073191107311467

Cattell, R. B. (1966). The Scree test for the number of factors. Multivariate Behavioral Research, 1(2), 245-276. doi: 10.1207/s15327906mbr0102_10

Cebolla, A., García-Palacios, A., Soler, J., Guillen, V., Baños, R., \& Botella, C. (2012). Psychometric properties of the Spanish validation of the Five Facets of Mindfulness Questionnaire (FFMQ). The European Journal of Psychiatry, 26(2), 118-126. doi:
10.4321/S0213-61632012000200005

Costello, A. B., \& Osborne, J. W. (2005). Best practices in exploratory factor analysis: Four recommendations for getting the most from your analysis. Practical Assessment, Research \& Evaluation 10(7). Recuperado de http://pareonline.net

Cronbach, L. J. (1951). Coefficient alpha and the internal structure of tests. Psychometrika, 16(3), 297-334. doi: 10.1007/BF02310555

De la Calle-Ortega, L. (2016). Psicología positiva. Propuesta de intervención en un aula de educación especial (Tesis de grado). Recuperado de http:// dehesa.unex.es/bitstream/handle/10662/3454/ TFGUEX_2015_Calle_Ortega.pdf?sequence=1

Diener, E. (1984). Subjective well-being. Psychological Bulletin, 95(3), 542-575. doi: 10.1037/00332909.95.3.542

Escurra-Mayaute, M., \& Salas-Blas, E. (2014). Construcción y validación del cuestionario de Adicción a Redes Sociales (ARS). LIBERABIT, 20(1), 73-91. Recuperado de http://revistaliberabit.com

Feldman, G., Hayes, A., Kumar, S., Greeson, J., \& Laurenceau, J. P. (2007). Mindfulness and emotion regulation: The development and initial validation of the Cognitive and Affective Mindfulness Scale-Revised (CAMS-R). Journal of Psychopathology and Behavioral Assessment, 29(3), 177-190. doi: 10.1007/s10862-006-9035-8

Feligreras-Alcalá, E. (2014). Técnicas de intervención en abuso sexual infantil: estrategias cognitivo-conductuales, aceptación y mindfulness (Tesis de grado). Recuperado de http://hdl.handle. net/10953.1/866

García-García, M. del M. (2015). Efecto de una intervención educativa mindfulness en los profesionales de un centro sociosanitario en La Rioja (Tesis de maestría). Recuperado de http://repositori.uji.es/ xmlui/handle/10234/153018

Greco, L., Baer, R., \& Smith, G. (2011). Assessing mindfulness in children and adolescents: Development and validation of the Child and Adolescent Mindfulness Measure (CAMM). Psychological Assessment, 23(3), 606-614. doi: 10.1037/a0022819.supp Grossman, P., Nieman, L., Schmidt, S., \& Walach, H. 
(2004). Mindfulness based stress reduction and health: A methaanalysis. Journal of Psychosomatic Research, 57(1), 35-43. doi: 10.1016/S00223999(03)00573-7

Hayes, A. M., \& Feldman, G. (2004). Clarifying the construct of Mindfulness in the context of emotion regulation and the process of change in therapy. Clinical Psychology: Science and Practice, 11(3), 225-262. doi: 10.1093/clipsy.bph080

Heidenreich, T., Ströhle, G., \& Michalak, J. (2006). Achtsamkeit: Konzeptuelle Aspekte und Ergebnisse zum Freiburger Achtsamkeitsfragebogen. Verhaltenstherapie, 16(1), 33-40. doi: $10.1159 / 000091521$

Hernando-Mazón, A. (2012). Atención plena y malestar psicológico: Un estudio piloto (Tesis de grado). Recuperado de http://zaguan.unizar.es/record/8287

Herndon, F. (2008). Testing mindfulness with perceptual and cognitive factors: External vs. internal encoding, and the Cognitive Failures Questionnaire. Personality and Individual Differences, 44(1), 3241. doi: 10.1016/j.paid.2007.07.002

Hervás, G., Cebolla, A., \& Soler, J. (2016). Intervenciones psicológicas basadas en mindfulness y sus beneficios: Estado actual de la cuestión. Clínica y Salud, 27(3), 115-124. doi: 10.1016/j.clysa.2016.09.002

Hofmann, S. G., Sawyer, A. T., Witt, A. A., \& Oh, D. (2010). The effect of mindfulness-based therapy on anxiety and depression: A meta-analytic review. Journal of Consulting and Clinical Psychology, 78(2), 169-183. doi: 10.1037/a0018555

Hu, L., \& Bentler, P. M. (1999). Cutoff criteria for fit indexes in covariance structure analysis: Conventional criteria versus new alternatives. Structural Equation Modeling, 6(1), 1-15. doi: 10.1080/10705519909540118

International Business Machines, IBM. (2013). IBM SPSS Statistics for Windows [Software]. Version 22.0. Armonk, NY: IBM.

Jiménez, S. S., Niles, B. L., \& Park, C. L. (2010). A mindfulness model of affect regulation and depressive symptoms: Positive emotions, mood regulation expectancies, and self-acceptance as regulatory mechanisms. Personality and Individual Differences,
49(6), 645-650. doi: 10.1016/j.paid.2010.05.041

Kabat-Zinn, J. (2013). Vivir con plenitud las crisis: Cómo utilizar la sabiduría del cuerpo y de la mente para afrontar el estrés, el dolor y la enfermedad ( $8^{\mathrm{a}} \mathrm{ed}$.). Barcelona: Kairós.

Kahneman, D. (2003). Objective happiness. En D. Kahneman, E. Diener \& N. Schwartz (Eds.), Well-being: The foundations of hedonic psychology (pp. 3-25). New York: Russell Sage Foundation.

Kaiser, H. F. (1960). The application of electronic computers to factor analysis. Educational and Psychological Measurement, 20(1), 141-151. doi: 10.1177/001316446002000116

Kaiser, H. F. (1970). A second generation Little Jiffy. Psychometrika, 35(4), 401-415. doi: 10.1007/ BF02291817

Kohls, N., Sauer, S., \& Walach, H. (2009). Facets of mindfulness - Results of an online study investigating the Freiburg Mindfulness Inventory. Personality and Individual Differences, 46(2), 224-230. doi: 10.1016/j.paid.2008.10.009

Laca-Arocena, F. A, Pérez-Verduzco, G., \& Vargas-Valencia, A. R. (2016). Multivariate analysis of the Spanish version of FMI-14: Contributions from the item response theory and biplot methods. $P A$ RIPEX - Indian Journal of Research, 5(10), 242245. doi: 10.15373/22501991

Lau, M. A., Bishop, S. R., Segal, Z. V., Buis, T., Anderson, N. D. ... Devins, G. (2006). The Toronto Mindfulness Scale development. Journal of Clinical Psychology, 62(12), 1445-1467. doi: 10.1002/ jclp.20326

Leigh, J., Bowen, S., \& Marlatt, G. A. (2005). Spirituality, mindfulness and substance abuse. Addictive Behaviors, 30(7), 1335-1341. doi: 10.1016/j.addbeh.2005.01.010

Lloret-Segura, S., Ferreres-Traver, A., Hernández-Baeza, A., \& Tomás-Marco, I. (2014). El análisis factorial exploratorio de los ítems: Una guía práctica, revisada y actualizada. Anales de Psicología, 30(3), 1151-1169. doi: 10.6018/analesps.30.3.199361

Marzo-Andreu, B. (2015). Estrés percibido y rasgo de mindfulness en el profesorado (Tesis de maestría). Recuperado de https://zaguan.unizar.es/re- 
$\operatorname{cord} / 31666 ?$

Pérula-Jiménez, C. (2016). Mindfulness en los profesionales de enfermería de atención primaria y su relación con el estrés laboral (Tesis de grado inédita). Universidad de Córdoba, España.

Quezada-Berumen, L. del C., González-Ramírez, M. T., Cebolla, A., Soler, J., \& García-Campayo, J. (2014). Conciencia corporal y mindfulness: Validación de la versión española de la escala de conexión corporal (SBC). Actas Españolas de Psiquiatría, 42(2), 57-67. Recuperado de https://www. actaspsiquiatria.es

Rubio-Galtier, I. (2015). Relación entre la capacidad de mindfulness de alumnos de bachiller con ansiedad, estrés y rendimiento académico (Tesis de maestría). Recuperado de https://zaguan.unizar.es/ record $/ 31667 ? \ln =$ en

Schmidt, C., \& Vinet, E. V. (2015). Atención plena: Validación del Five Facet Mindfulness Questionnaire (FFMQ) en estudiantes universitarios chilenos. Terapia Psicológica, 33(2), 93-102. doi: 10.4067/ S0718-48082015000200004

Segal Z. V., Williams, J. M. G., \& Teasdale, J. D. (2012). Mindfulness-based cognitive therapy for depression: A new approach to preventing relapse $\left(2^{\mathrm{a}}\right.$ ed.). New York: Guilford.

Seligman, M. (1992). Learned optimism. New York: Pocket Books Simon Schuster Inc.

Siegel, R. D., Germer, C. K., \& Olendzki, A. (2009). Mindfulness: What is it? Where did it come from? En Didonna, F. (Ed.), Clinical Handbook of Mindfulness. New York: Springer.

Simón, V. (2013). Aprender a practicar mindfulness $\left(8^{\mathrm{a}}\right.$ ed.). Madrid: Sello Editorial.

Sociedad Mexicana de Psicología. (2007). Código Ético del Psicólogo. México: Trillas.

Soler, J. (2014). Evaluación del mindfulness. En A. Cebo1la, J. García-Campayo, \& M. Demarzo (Coords.), Mindfulness y ciencia: De la tradición a la modernidad (cap. 2, pp. 41-63). Madrid: Alianza Editorial.

Soler, J., Tejedor, R., Feliu-Soler, A., Pascual, J. C., Cebolla, A., Soriano, J., Alvarez, E., \& Perez, V. (2012). Propiedades psicométricas de la versión española de la escala Mindful Attention Awareness Scale (MAAS). Actas Españolas de Psiquiatría, 40(1), 19-26. Recuperado de https://www.actaspsiquiatria.es

Tejedor, R., Feliu-Soler, A., Pascual, J. C., Cebolla, A., Portella, M. J., Trujols, J. ... Soler, J. (2014). Propiedades psicométricas de la versión española de la Philadelphia Mindfulness Scale. Revista de Psiquiatría y Salud Mental, 7, 157-65. doi: 10.1016/j. rpsm.2014.04.001

Torné-Novell, M. (2013). Mindfulness y musicoterapia (Tesis de maestría). Universitat de Vic, Instituto Superior de Estudios Psicológicos, España.

Trousselard, M., Steiler, D., Raphel, C., Cian, C., Duymedjian, R., Claverie, D., \& Canini, F. (2010). Validation of a French version of the Freiburg Mindfulness Inventory - Short version: Relationships between mindfulness and stress in an adult population, Biopsychosocial Medicine, 4(1), 8. doi: 10.1186/1751-0759-4-8

Turanzas, J. (2013). Adaptación transcultural de la escala CAAM (Child and Adolescent Mindfulness Measure) (Tesis de maestría). Universidad Internacional Valenciana, España.

Walach, H., Buchheld, N., Buttenmüller, V., Kleinknecht, N., \& Schmidt. S. (2006). Measuring mindfulness - the Freiburg Mindfulness Inventory (FMI). Personality and Individual Differences, 40(8), 1543-1555. doi: 10.1016/j.paid.2005.11.025

Williams, B., Onsman, A., \& Brown, T. (2010). Exploratory factor analysis: A five-step guide for novices. Australasian Journal of Paramedicine, 8(3), 1-13. Recuperado de https://ajp.paramedics.org 


\section{Anexo \\ Inventario Friburgo de Mindfulness (IFM-14) \\ (Traducción y validación del Freiburg Mindfulness Inventory-FMI-14- de Walach, Buchheld, Buttenmüller, Kleinknecht, \& Schmidt, 2006)}

Instrucciones: A continuación, encontrará descripciones de diferentes situaciones personales, indique encerrando en un círculo en cada una de ellas con qué frecuencia suceden en su caso.

1: Raramente 2: Ocasionalmente 3: Con frecuencia 4: Casi siempre

1. Estoy abierto a experimentar el momento presente.

2. Siento mi cuerpo cuando cocino, limpio o hablo con otras personas.

3. Cuando me doy cuenta de que mi mente está distraída, suavemente regreso a la experiencia de aquí y ahora.

4. Soy capaz de apreciarme a mí mismo.

5. Presto atención a lo que hay detrás de mis acciones.

6. Veo mis errores y dificultades sin juzgarlos.

7. Me siento conectado con mi experiencia del aquí y el ahora.

8. Acepto las experiencias desagradables.

9. Soy amable conmigo mismo cuando las cosas van mal.

10. Observo mis sentimientos sin perderme en ellos.

11. En situaciones difíciles, puedo hacer una pausa sin reaccionar inmediatamente.

12. Experimento momentos de paz interior y tranquilidad aún cuando las cosas se pongan agitadas y estresantes. 